\title{
Burkina-Faso : petites interrogations a propos d'une lecture
}

\author{
Yannick Jaffré et Alain Demaison
}

\section{(2) OpenEdition}

\section{Journals}

Édition électronique

URL : http://journals.openedition.org/apad/354

DOI : 10.4000/apad.354

ISSN : 1950-6929

Éditeur

LIT Verlag

\section{Édition imprimée}

Date de publication : 15 décembre 1991

\section{Référence électronique}

Yannick Jaffré et Alain Demaison, « Burkina-Faso : petites interrogations a propos d'une lecture », Bulletin de l'APAD [En ligne], 2 | 1991, mis en ligne le 21 juillet 2006, consulté le 08 septembre 2020 URL : http://journals.openedition.org/apad/354; DOI : https://doi.org/10.4000/apad.354

Ce document a été généré automatiquement le 8 septembre 2020

Bulletin de l'APAD 


\title{
Burkina-Faso : petites interrogations a propos d'une lecture
}

\author{
Yannick Jaffré et Alain Demaison
}

1 Dans le précédent numéro du bulletin de l'A.P.A.D, Doris Bonnet nous présente un "état des lieux" de l'anthropologie appliquée à des problèmes de développement au Burkina Faso. Elle évoque à ce propos les enquêtes qui furent menées dans le cadre du projet "F.T.C." ("Formation Technique Continue dans cinq provinces de l'ouest Burkina"). Nous l'en remercions, et souhaitons, dans cette courte note, prolonger les questions implicitement posées par son texte.

2 Celles-ci nous semblent être, en effet, au centre du débat entre "développeurs", ou "anthropologues impliqués", et "anthropologues chercheurs" et concerner non seulement les critères de choix des objets de la recherche mais aussi les modalités de sa pratique. Pour ce faire, il nous semble important de préciser brièvement la méthodologie que nous avons employée dans notre projet, qui, bien qu'étant de type ethnolinguistique, ne s'y limitait pas.

3 En effet, s'il nous était nécessaire de disposer d'un savoir sur les terminologies et les pratiques concernant l'élevage ou les systèmes de gestion afin d'en avoir une meilleure compréhension, l'objet de notre travail était avant tout de permettre l'instauration d'un réel dialogue entre des techniciens et des populations rurales. L'enquête, dans ce cadre, n'avait plus pour simple but de recenser des savoirs populaires, mais surtout d'entraîner un questionnement des implicites des discours techniques par leurs utilisateurs, les divers agents de développement.

4 Pour ce faire, ce travail respectait plusieurs étapes toutes menées avec les techniciens : 1) Élaboration d'un questionnaire d'enquête en langue française (la langue de travail reste le français) ; 2) Traduction du questionnaire en langue vernaculaire. Les langues de travail sont ici le français et le dioula afin que la compréhension des termes se fasse par variation sémantique interne aux deux idiomes avant de l'être par traduction ; 3) Réalisation de l'enquête par les techniciens ; 4) Dépouillement de l'enquête et rédaction 
des textes de formation en langue nationale. La langue de référence et de travail est le dioula ; 5) Publication des textes ; 6) Mise en place et animation des formations par les techniciens des différents secteurs d'activité.

Ces travaux de recherches ont, bien évidemment permis de recueillir de nombreuses informations, mais surtout ils ont permis de rédiger 21 textes d'alphabétisation publiés à 2500 exemplaires, servant de supports pédagogiques pour 200 formations regroupant pour une année 2000 paysans et éleveurs ainsi que 150 techniciens et encadreurs formés à cette démarche.

6 Tout autant qu'un recensement des connaissances des populations rurales en vue de les analyser ou de les améliorer, ces enquêtes puis ces formations ont entraîné une modification du rapport des techniciens à leur savoir, celui-ci n'ayant plus l'évidence d'un dogme mais apparaissant comme "un point de vue spécifique sur le monde". Ils ont ainsi pu mesurer les difficultés liées à l'articulation des discours techniques régis à juste titre par certaines règles scientifiques et des "savoirs populaires", liés à d'autres contraintes. Nous n'approfondirons pas plus ce sujet renvoyant ceux qui seraient intéressés au livre "D'un savoir à l'autre" (J.-P.olivier de Sardan \& E. Paquot. éd. Documentation française, Paris : 1991) (cf. rubrique "Lu et à lire").

7 Sur un plan plus général, cette expérience d'anthropologie pratique, par sa différence avec des travaux plus classiques pose quelques problèmes que nous ne ferons ici qu'évoquer.

8 Le premier concerne la nécessité d'un échantillonnage des populations auprès desquelles nous travaillons. Celui-ci peut être quantitatif et s'inspirer des techniques de l'épidémiologie, ou qualitatif, croisant les positions sociales avec la diversité des énoncés. Que l'on choisisse l'une ou l'autre de ces possibilités, il est nécessaire de le préciser afin qu'une parole singulière n'en vienne à représenter, par une "sournoise extension" l'expression d'un groupe. Comme dans les sciences "dures", l'opposition n'est pas entre vrai ou faux mais entre signifiant et insignifiant. Un énoncé n'est jamais faux mais il peut être parfaitement insignifiant rapporté à un ensemble social.

9 Si l'anthropologie du développement a, ne serait-ce que modestement, pour but de modifier le réel, elle ne peut éviter cette question de la représentativité et de la pertinence des énoncés recueillis sous peine de ne s'adresser aux populations ou de n'élaborer des projets qu'à partir de fictions. Le romanesque et l'assentiment de ses pairs sont parfois suffisants au discours universitaires, ils ne sauraient l'être pour ceux qui sont confrontés à divers groupes sociaux dans des programmes de développement.

10 Le deuxième point concerne la formation des anthropologues "du développement". Les découpages universitaires sont-ils pertinents ou faut-il envisager, sans négliger l'apprentissage des disciplines de base, d'autres formations plus adaptées aux problèmes à résoudre? Par exemple les anthropologues "classiques" sont souvent capables d'agrémenter leurs textes de quelques mots pris dans des langues nationales (ah ! le "banmana"...mais pourquoi pas le "english"!). mais bien souvent incapables de dialoguer avec les locuteurs de leur "terrain" ou de décrire sérieusement un champ sémantique (ah! les effets d'exotisme obtenus par des citations hors contexte!). Ne faudrait-il pas alors accentuer des programmes d'apprentissages de langues? De même, peut-on sérieusement faire actuellement de l'anthropologie rurale ou de la santé sans connaître un minimum d'agronomie, de santé publique et d'économie? Ne risque-t-on pas dans ce cas de prendre des effets pour des causes et attribuer par exemple la mauvaise utilisation des systèmes sanitaires à diverses représentations sociales au lieu 
de considérer plus simplement le manque de médicaments et l'attitude des personnels de santé ?

11 Les praticiens du développement ne pourraient-ils aider à la définition et à ranimation scientifique de ces nouveaux cursus qui pourraient être conçus non plus seulement en fonction de la position des diverses disciplines dans le champ du savoir mais en fonction d'une analyse des tâches qui attendent le futur anthropologue intéressé par les problèmes du développement (ceux-ci ne se limitant bien évidemment pas uniquement aux pays du sud, mais concernant par exemple les questions de l'émigration)?

Le troisième point concerne la validation des travaux. Celle-ci s'effectue le plus souvent selon des critères de cohérence discursive de thèses ou de publications. Sans confondre les anthropologues et les assistants sociaux, le problème reste posé d'une vérification complémentaire de la pertinence d'une hypothèse par sa mise en œuvre efficace dans une pratique. Celle-ci ne peut se confondre avec l'expérience du "terrain", qui n'est souvent qu'un prolongement "physique" des débats théoriques, mais pourrait peut-être prendre la forme, plus impliquée ou politique, d'un projet. Il s'agirait dans ce cas d'éprouver la validité d'un travail en fonction de sa capacité à aider concrètement à une meilleure prise en compte des "identités" et des "demandes" des usagers du développement.

13 Peut-on cumuler ces deux démarches ou faut-il distinguer des "anthropologues du développement" et des "anthropologues dans le développement" ? Pour notre part sans réponse, nous espérons que ces quelques réflexions, au fil d'une lecture, sauront solliciter d'autres plumes afin que s'épanouissent de nouvelles connaissances alliant la rondeur des savoirs conséquents au tranchant de l'action.

\section{AUTEURS}

\section{ALAIN DEMAISON}

Niamey-Bobo Dioulasso 